\title{
Immune-Therapy Standards Of Care
}

\author{
Frank Griesinger* \\ Department of Hematology and Oncology, University Internal Medicine-Oncology, Germany
}

Submission: September 10, 2019; Published: October 10, 2019

*Corresponding author: Frank Griesinger, Department of Hematology and Oncology, university Internal Medicine-Oncology, Pius-Hospital, University Medicine of Oldenburg, Germany

Keywords: NSCLC; Immune-Checkpoint-Inhibitors; Metastatic diesease; Stage IV; Advanced disease; Stage III

\section{Introduction}

Lung cancer ist the most frequent deadly malignant disease in men and the second most deadly malignant disease in women [1]. Two "leap" innovations, the detection of driver mutations in about $25 \%$ of patients and the observation that NSCLC is an immune-checkpoint-inhibitor responsive disease leading to a dramatic improvement in ORR, PFS and OS, therapeutic standards in NSCLC have changed dramatically over the past decade [2]. These dramatic changes in outcome can only be achieved if patients are tested for the relevant "biomarkers" , i.e. genetic alterations EGFR, ALK, ROS, BRAF (mandatory) and PD-L1 before the start of treatment to allow an optimal 1st line treatment stratification [3-6]. This article describes the treatment strategies in patients with NSCLC without driver mutations.

\section{NSCLC without driver mutations}

Patients that do not show any driver mutations i.e. EGFR and ALK WT were included in trials that studied immune checkpoint inhibitors. WT definition should be expanded most likely to ROS, HER2, MET and RET, whether BRAF mt+ positive patients might be more responsive to ICI will have to elucidated. Patients with driver muations (EGFR, ALK, ROS; BRAF, RET, MET) should be treated with TKI, only when TKI treatment is exhausted, ICI potentially in combination with chemotherapy should be employed.

\section{PD-L1 expression $>\mathbf{5 0 \%}$ without driver mutation}

Patients with a PD-L1 expression of $>50 \%$ (about $30 \%$ of the total NSCLC population) can be treated with pembrolizumab single agent therapy on the basis of the KN 24 study[7] leading to a median OS of 30 months, while it was 14 months with chemotherapy (HR OS 0.63). PFS, ORR (28vs. $48 \%$ ) and QoL were also significantly improved with Pembrolizmab single agent. Test rates have improved in Germany from 20 to $75 \%$, however not every patient that is tested will receive Pembrolizumab in 1st line [6]. On the basis of KN 189 and KN 407 studies, patients with a high PD-L1 expression are also amenable for a combined ICI and chemotherapy treatment.

\section{ICI-Chemotherapy combination in non-squamous cell carcinoma without driver mutations independent of PD-L1 expression}

In the KN 189 study [8] patients with non-squamous cell carcinoma without driver mutations (EGFR and ALK) were included independently on the PD-L1 status, patients were stratified for PD-L1 expression $>1 \%$ and $<1 \%$. The median OS was significantly improved over control chemotherapy with a HR of 0.42 , the OS benefit was seen in all PD-L1 subgroups. This study showed for the first time a synergistic effect of chemotherapy and ICI even in PD-L1 negative tumors. At ASCO 2019, PFS was updated for the study, and there was also a statistically significant PFS benefit shown for the PD-L1 0\% subgroup. The combination therapy of Pembrolizumab, Pemetrexed and Cis- or Carboplatin has been approved since August 2018 by the EMA. The second approved combination in non squamous cell NSCLC is the IMPOWER 150 regimen with Paclitaxel, Carboplatin, Bevacizumab and Atezolizumab [9]. The overall study was positive for the endpoint OS, although this was mainly driven by the OS benefit in the PD-L1 TC3/IC3 (high PD-L1 expression) group. However, two specific signals could be derived from the Impower study: 1. Patients with liver metastases benefitted from the quadruple therapy with a HR of 0,52 for OS, while the HR for the complete study was only 0.73 . There is some preclinical data to support the antiangiogenic effect in the liver environment. The data in the IMOWER study on liver metastases are of high quality as liver metastases were a prespecified stratification parameter. 2. Patients with EGFR and ALK alterations were included in this trial. For patients were TKI therapy was exhausted, a significant OS benefit was seen for the Pacli-Carbo-Bev -Atezo therapy over the Sandler standard (pacli-carbo-bev). This was seen in 
the group of patients that had been treated with TKI as prior therapy with a HR of 0.54 , and in the patient group with common mutations up to 0.32 . Therefore, this combination should be the preferred choice for patients with exhaustion of TKI therapy and potentially for patients with liver metastases.

\section{ICI-Chemotherapy-combinations in squamous cell carcinoma independent of PD-L1 expression}

The licensing study of chemo-ICI therapy in squamous cell carcinoma is the KN 407 [10], which included all comer squamous cell carcinomas. Paclitaxel or nab-Paclitaxel/ Carboplatin +/- Pembrolizumab were compared. Patients were stratified according to PD-L1 expression $(<1 \%,>1 \%)$. In all subgroups, a statistically significant advantage of the coprimary endpoints OS and PFS was seen, HR für PFS und OS were 0.56 and 0.64 respectively. Therefore patients with squamous cell carcinoma should receive ICI + chemotherapy as a standard of care. Approval was given in March 2019 for this combination by the EMA.

\section{ICI chemo-combination vs. ICI single agent therapy in patients with PD-L1 expression > 50\%}

The question, whether patients with a high PD-L1 expression of $>50 \%$ should be treated with the combination (chemo+ICI) or single agent ICI has not been addressed in a head to head randomized clinical trial. A meta-analysis has recently been published in the Public Domain (GBA AMNOG Dossier of MSD [11], that compared pembro single agent against chemo + pembrolizumab for non-squamous cell carcinomas and for squamous cell carcinomas with a PD-L1 expression of $>50 \%$. The data show a highly significant advantage for Chemo- + ICI therapy in non-squamous cell carcinoma (HR 0.4, p=0,008). The rate of side effects and the QoL were not significantly increased by the combination. Exceptions were alopecia and social competence. In contrast, patients with squamous cell carcinoma (PD-L1 $>50 \%$ ) in the indirect comparison between Chemo-ICI vs. ICI with a HR of 1.06. The adverse events were significantly higher with a OR of $2.85(\mathrm{p}=0,009)$. This meta-analysis could be helpful in deciding between chemo-ICI and ICI-single agent in PD-L1 high expressers. In conclusion, combination therapy most likely should be preferred in non-squamous cell carcinoma, especially in patients with high tumor burden and high remission pressure, and most likely in patients with never or light smoking status (HR $0.9,95 \%$ CI 0.11-7.59). Patients with squamous cell carcinoma should be rigorously tested for fitness for chemotherapy and might be better off with single agent pembrolizumab based on the meta-analysis. Although ICI are a cornerstone of therapy of metastatic NSCLC and has been denominated the 5th column of cancer therapy, it is not yet a standard of care in early and advanced stage NSCLC. The only exception is the administration of Durvalumab in stage III NSCLC after radio-chemotherapy, having responded and not experiencing radiotherapy-induced pneumonitis. Durvalumab has been approved by the EMA based on the PACIFIC trial, that included patients only after radiochemotherapy. Recently, the 3 year OS data were shown at ASCO showing a survival rate of 57.0 (Durvalumab) vs. $43.5 \%$ (control arm), HR 0.69 . Relative to the PD-L1 > 1\% (approval by the EMA), the HR for OS was 0.59. Therefore, patients that are treated with radio-chemotherapy should be tested for PD-L1 and should be treated in case of a PD-L1 expression of $>1 \%$ with durvalumab, if possible not much later than 2 weeks after the end of radiochemotherapy.

\section{References}

1. https://www.uicc.org/new-global-cancer-data-globocan-2018

2. https://www.awmf.org/uploads/tx_szleitlinien/020-0070L_l_S3_ Lungenkarzinom_2018-03.pdf

3. Junker K, Büttner R, Langer T, Ukena D (2018) Pathological-anatomical diagnosis according to the German lung cancer guideline 2018. Pathologe 39(6): 589-603.

4. Eberhardt WE, Mitchell A, Crowley J, Kondo H, Kim YT, et al. (2015) The IASLC Lung Cancer Staging Project: Proposals for the Revision of the M Descriptors in the Forthcoming Eighth Edition of the TNM Classification of Lung Cancer. J Thorac Oncol 10(11): 1515-1522.

5. https://www.onkopedia.com/de/onkopedia/guidelines/ lungenkarzinom-nicht-kleinzellig-sclc/@@view/html/index.html

6. Frank G, Wilfried Ernst EE, Arnd N, Marcel R, Mark-Oliver Z, et al. (2018) Molecular testing, frequency of molecular alterations and targeted 1st-line treatment of patients with non-small cell lung carcinoma in Germany. Results from the prospective German Registry CRISP (AIOTRK-0315): Journal of Clinical Oncology 36(15).

7. Reck M, Rodríguez-Abreu D, Robinson AG, Hui R, Csőszi T, et al. (2016) Pembrolizumab versus Chemotherapy for PD-L1-Positive Non-SmallCell Lung Cancer. N Engl J Med 375(19): 1823-1833.

8. Gandhi L, Rodríguez-Abreu D, Gadgeel S, Esteban E, Felip E, et al. (2018) Pembrolizumab plus Chemotherapy in Metastatic Non-SmallCell Lung Cancer. N Engl J Med 378(22): 2078-2092.

9. Socinski MA, Jotte RM, Cappuzzo F, Orlandi Stroyakovskiy FD, Nogami N, et al. (2018) Atezolizumab for First-Line Treatment of Metastatic Nonsquamous NSCLC: N Engl J Med 378(24): 2288-2301.

10. Paz-Ares L, Luft A, Vicente D, Tafreshi A, Gümüş M, et al. (2018) Pembrolizumab plus Chemotherapy for Squamous Non-Small-Cell Lung Cancer. N Engl J Med 379(21): 2040-2051.

11. Antonia SJ, Villegas A, Daniel D, Vicente D, Murakami S, et al. (2018) Overall Survival with Durvalumab after Chemoradiotherapy in Stage III NSCLC. N Engl J Med 379(24): 2342-2350. 
Your next submission with Juniper Publishers will reach you the below assets

- Quality Editorial service

- Swift Peer Review

- Reprints availability

- E-prints Service

- Manuscript Podcast for convenient understanding

- Global attainment for your research

- Manuscript accessibility in different formats (Pdf, E-pub, Full Text, Audio)

- Unceasing customer service

Track the below URL for one-step submission https://juniperpublishers.com/online-submission.php 